\title{
Ensemble Inequivalence in Mean-field Models of Magnetism
}

\author{
Julien Barré1,3, David Mukamel ${ }^{2}$, and Stefano Ruffo ${ }^{1,3}$ \\ 1 Ecole Normale Supérieure de Lyon, Laboratoire de Physique, 46 Allée d' Italie, \\ 69364 Lyon Cedex 07, France \\ 2 Department of Physics of Complex Systems, The Weizmann Institute of Science, \\ Rehovot 76100, Israel \\ 3 Dipartimento di Energetica "Sergio Stecco", Università di Firenze, via s. Marta 3 \\ 50139 Firenze, Italy, INFM and INFN.
}

\begin{abstract}
Mean-field models, while they can be cast into an extensive thermodynamic formalism, are inherently non additive. This is the basic feature which leads to ensemble inequivalence in these models. In this paper we study the global phase diagram of the infinite range Blume-Emery-Griffiths model both in the canonical and in the microcanonical ensembles. The microcanonical solution is obtained both by direct state counting and by the application of large deviation theory. The canonical phase diagram has first order and continuous transition lines separated by a tricritical point. We find that below the tricritical point, when the canonical transition is first order, the phase diagrams of the two ensembles disagree. In this region the microcanonical ensemble exhibits energy ranges with negative specific heat and temperature jumps at transition energies. These two features are discussed in a general context and the appropriate Maxwell constructions are introduced. Some preliminary extensions of these results to weakly decaying nonintegrable interactions are presented.
\end{abstract}

\section{Introduction}

Thermodynamics of systems with long range interactions is quite distinct from that of systems where the interactions are short ranged. For pairwise interactions, long range potentials decay at large distances as $V(r) \sim 1 / r^{\alpha}$ with $0 \leq \alpha \leq d$ in $d$ dimensions. Such systems are not extensive, although models used to describe them can be made extensive by an appropriate volume dependent rescaling (the Kac prescription) [1] . However, even with such rescaling, these models remain inherently non-additive. Namely, when divided into two or more macroscopic subsystems, the total energy (or other extensive quantity) of the system, is not necessarily equal to the sum of the energies of the subsystems. Since additivity is an essential ingredient in the derivation of thermodynamics and statistical mechanics of systems with short range interactions, its violation may lead to interesting and unusual effects.

To illustrate the lack of additivity in these systems consider an extremely simple example of an Ising model with infinite range, mean-field like, interactions, 
corresponding to $\alpha=0$. The Hamiltonian takes the form

$$
H=-\frac{J}{2 N}\left(\sum_{i=1}^{N} \sigma_{i}\right)^{2}
$$

where $\sigma_{i}= \pm 1$ is the spin $1 / 2$ variable on site $i$ and $N$ is the number of spins in the system. This Kac type pre-factor is taken to ensure extensivity. Let us consider for simplicity a system with vanishing magnetization, $M=\sum_{i=1}^{N} \sigma_{i}=$ 0 . It is clear that the energy, $E=H$, of the system satisfies $E=0$. Now let us divide the system into two subsystems, $I$ and $I I$, each composed of $N / 2$ sites, where all spins in subsystem $I$ are up while those in subsystem $I I$ are down (see Fig. 1). The energies of the two subsystems, $E_{I}$ and $E_{I I}$ satisfy $E_{I}=E_{I I}=$ $-J N / 4$. Since the sum of the two energies $E_{I}+E_{I I}$ is not equal to the total energy $E$ the system is clearly not additive.



Fig. 1. A two phase configuration of the infinite range interaction Ising model for which the energy is non-additive

The lack of additivity may result in many unusual properties of systems with long range interactions, like inequivalence of various ensembles, negative specific heat in the microcanonical ensemble and possible temperature discontinuity at first order transitions. For example, the usual argument for the non-negativity of the specific heat, or equivalently, for the concavity of the entropy-energy curve $s(\epsilon)$, makes use of the fact that systems with short range interactions are additive. Here $s=S / N$ and $e=E / N$ are the entropy and energy per particle, respectively. An entropy curve which is not concave in the energy interval $e_{1}<e<e_{2}$ (see Fig. 2) is unstable for systems with short range interactions since entropy may be gained by phase separating the system into two subsystems with energy densities $e_{1}$ and $e_{2}$ while keeping the total energy fixed. In particular the average energy 
and entropy densities in the coexistence region is given by the weighted average of the corresponding densities of the two coexisting systems, and thus the correct entropy curve in that region is given by the common tangent line (see Fig. 2), resulting in an overall concave curve. On the other hand for systems with long range interactions, additivity does not hold, and thus it is not always the case that entropy may be gained by phase separation. In such cases the non-concave entropy curve may in fact be the correct entropy of the system, resulting, for example, in negative specific heat.

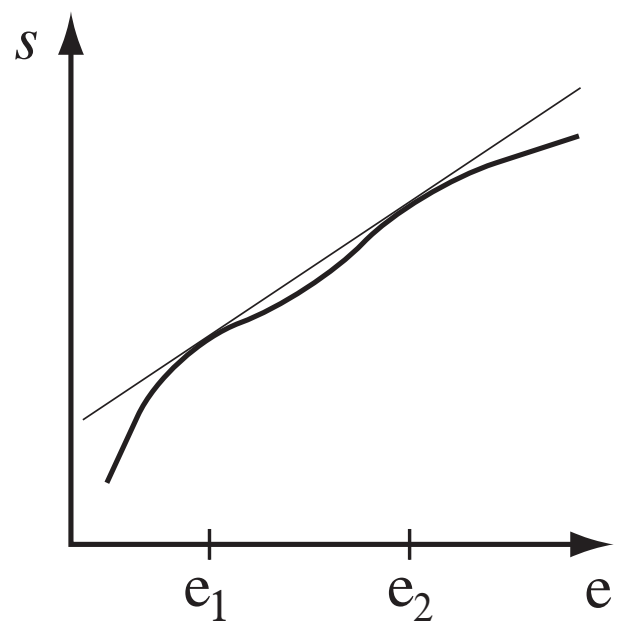

Fig. 2. A non-concave entropy curve, which for additive systems is made concave by considering two phase coexistence configurations in the region where the entropy of the homogeneous system is non-concave. This procedure is not applicable for non-additive systems like those with long range interactions.

Self gravitating particles are perhaps the most extensively studied systems with long range forces (corresponding to $\alpha=1$ in $d=3$ dimensions). The fact that the entropy of these systems is not necessarily a concave function of the energy has first been pointed out by Antonov [2]. The thermodynamical consequences of this observation have been elaborated using simple models by Lynden-Bell [3], Thirring [4] and others (see also the papers by Chavanis [5] and Padhmanaban [6] in this book for a review).

In fact, non-additivity is not limited to systems with long range interactions. Finite systems with short range interactions, such as atomic clusters, are nonadditive as long as they are small enough so that surface energy may not be neglected as compared with the bulk energy [7]. Negative specific heat in clusters of atoms has been discussed by R. M. Lynden-Bell [8 and it has been recently observed experimentally, as discussed in this book in Refs. [7].9]. 
In the present paper we consider a simple spin 1 mean-field model $(\alpha=0)$ and study its phase diagram both within the canonical and the microcanonical ensembles. This model, known as the Blume-Emery-Griffiths (BEG) model [10], may serve as a useful tool for studying thermodynamic effects which characterize systems with long range interactions. The canonical phase diagram of this model has been studied in the past, and it exhibits a line of phase transitions separating a magnetically ordered phase from a paramagnetic one. The line is composed of a first order transition and a second order transition segments separated by a tricritical point. The microcanonical phase diagram on the other hand exhibits some rather distinct features 11. While the second order segment of the canonical phase diagram is present also in the microcanonical ensemble, the nature of the first order segment, its location and the location of the tricritical point are rather different. In this ensemble, a region with negative specific heat is found, as well as a first order transition at which the temperatures of the two coexisting phases are different.

The paper is organized as follows: in Sec. 2 the BEG model is introduced and its canonical phase diagram is studied. The microcanonical phase diagram and the relationship between the two ensembles are studied in Sec. 3. Some features of the Maxwell constructions leading to temperature jumps are presented in Sec. 因. An alternative more general derivation of the expression for microcanonical entropy is presented in Sec. 5 . The results presented in this paper are generalized to models with slowly decaying interactions (with $0<\alpha<d$ ) in Sec. 6. Finally, concluding remarks are given in Sec. 7

\section{The Blume-Emery-Griffiths model in the canonical ensemble}

We consider a spin model with infinite range, mean-field like, interactions whose phase diagram can be analyzed analytically both within the canonical and the microcanonical ensembles. This study enables one to compare the two resulting diagrams and get a better understanding of the effect of the non-additivity on the thermodynamic behavior of the system. The model we consider is a simple version of the Blume-Emery-Griffiths (BEG) model [10], known as the BlumeCapel model, with infinite range interactions. The model is defined on a lattice, where each lattice point $i$ is occupied by a spin- 1 variable $S_{i}=0, \pm 1$. The Hamiltonian is given by

$$
H=\Delta \sum_{i=1}^{N} S_{i}^{2}-\frac{J}{2 N}\left(\sum_{i=1}^{N} S_{i}\right)^{2}
$$

where $J>0$ is a ferromagnetic coupling constant and $\Delta$ controls the energy difference between the magnetic $\left(S_{i}= \pm 1\right)$ and the non-magnetic $\left(S_{i}=0\right)$ states. The canonical phase diagram of this model in the $(T, \Delta)$ plane has been studied in the past [10]. The free energy

$$
f(\beta)=-\frac{1}{\beta} \lim _{N \rightarrow \infty} \frac{\ln Z}{N},
$$


where $Z$ is the partition function

$$
Z=\sum_{\left[S_{i}\right]} \exp -\beta H
$$

with $\beta=1 / k_{B} T$ and $k_{B}$ the Boltzmann constant, can be exactly derived in the $N \rightarrow \infty$ limit. One uses the Hubbard-Stratonovich transformation, which in this case amounts to the simple Gaussian identity

$$
\exp \left(b a^{2}\right)=\sqrt{\frac{b}{\pi}} \int_{-\infty}^{\infty} d x \exp \left(-b x^{2}+2 a b x\right),
$$

with $a=\sum_{i} S_{i} / N$ and $b=\beta J N / 2$. One then easily gets

$$
Z=\sqrt{\frac{\beta J N}{2 \pi}} \int_{-\infty}^{\infty} d x \exp (-N \beta \tilde{f}(\beta, x))
$$

where

$$
\beta \tilde{f}(\beta, x)=\frac{1}{2} \beta J x^{2}-\ln \left[1+e^{-\beta \Delta}\left(e^{\beta J x}+e^{-\beta J x}\right)\right] .
$$

Using Laplace's method to perform the integral in formula (6) in the $N \rightarrow \infty$ limit, one finally gets

$$
f(\beta)=\min _{x} \tilde{f}(\beta, x)
$$

At $T=0$ the model has a ferromagnetic phase for $2 \Delta / J<1$ and a non-magnetic phase otherwise separated by a first order phase transition. Indeed, the paramagnetic zero temperature state $S_{i}=0, \forall i$ is degenerate with the ferromagnetic state $S_{i}=1, \forall i$ (or $S_{i}=-1, \forall i$ ) at $2 \Delta / J=1$ (this latter state being the ground state for $2 \Delta / J<1)$, a typical scenario for first order phase transitions. At $\Delta=-\infty$ the model reduces to the mean-field Ising model and hence it has a second order phase transition at $k_{B} T / J=1$. The $(T, \Delta)$ phase diagram displays a transition line separating the low temperature ferromagnetic phase from the high temperature paramagnetic phase. The transition line is found to be first order at high $\Delta$ values, while it is second order at low $\Delta$. The second order line is found by locating the instability of the paramagnetic phase $x=0$, which means finding the condition for the $x^{2}$ coefficient in formula (7) to be zero (note that $\tilde{f}(\beta, x)$ is even in $x)$. One gets for the second order term

$$
\beta J=\frac{1}{2} e^{\beta \Delta}+1 .
$$

The two segments of the transition line (high and low $\Delta$ ) are separated by a tricritical point located at $\Delta / J=\ln (4) / 3 \simeq 0.4621, \beta J=3$. This is obtained by requiring that both the $x^{2}$ coefficient of $\tilde{f}(\beta, x)$ in (7) and the $x^{4}$ coefficient

$$
\frac{1}{3}-\frac{2 \exp (-\beta \Delta)}{(1+2 \exp (-\beta \Delta)}
$$


vanish. The first order segment of the transition line is obtained numerically by equating the free energies of the ferromagnetic and the paramagnetic states. The behavior of the function $\beta \tilde{f}(\beta, x)$ as $\beta$ varies is shown in Fig. 3 at a second order phase transition $(\Delta=0)$ and in Fig. 1 at a first order phase transition $(\Delta=0.476190)$.

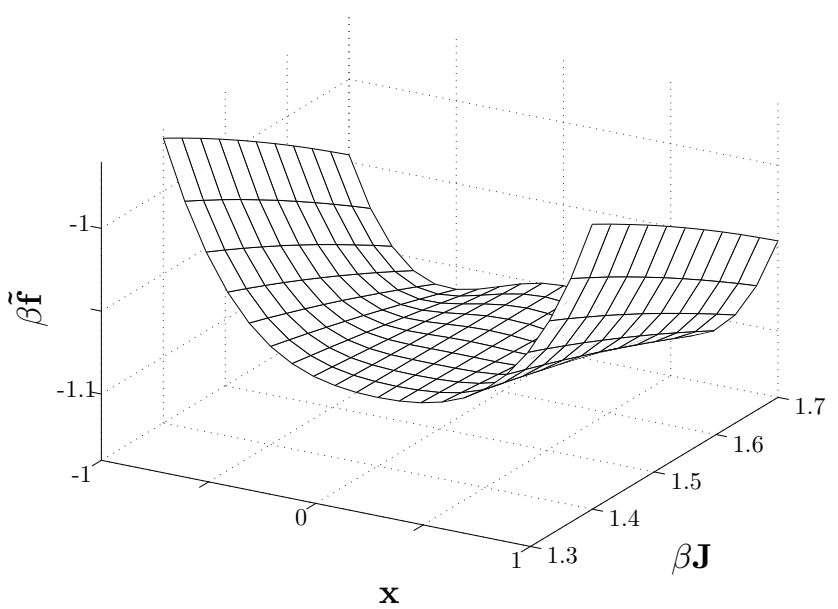

Fig. 3. $\beta \tilde{f}(\beta, x)$ as a function of $\beta J \in[1.4,1.7]$ and $x \in[-1,1]$ for $\Delta=0$. The second order transition is at $\beta J=3 / 2$ where the minimum at $x=0$ becomes a maximum and two side minima develop

\section{Microcanonical solution of the Blume-Emery-Griffiths model}

The derivation of the phase diagram of the BEG model (2) in the microcanonical ensemble reduces to a simple counting problem, since all spins interact with equal strength, independently of their mutual distance (there is no space in the model). A given microscopic configuration is characterized by the numbers $N_{+}, N_{-}, N_{0}$ of up, down and zero spins, with $N_{+}+N_{-}+N_{0}=N$. The energy $E$ of this configuration is only a function of $N_{+}, N_{-}$and $N_{0}$ and is given by

$$
E=\Delta Q-\frac{J}{2 N} M^{2}
$$

where $Q=\sum_{i=1}^{N} S_{i}^{2}=N_{+}+N_{-}$(the quadrupole moment) and $M=\sum_{i=1}^{N} S_{i}=$ $N_{+}-N_{-}$(the magnetization) are the two order parameters. The number of microscopic configurations $\Omega$ compatible with the macroscopic occupation numbers $N_{+}, N_{-}$and $N_{0}$ is

$$
\Omega=\frac{N !}{N_{+} ! N_{-} ! N_{0} !} .
$$




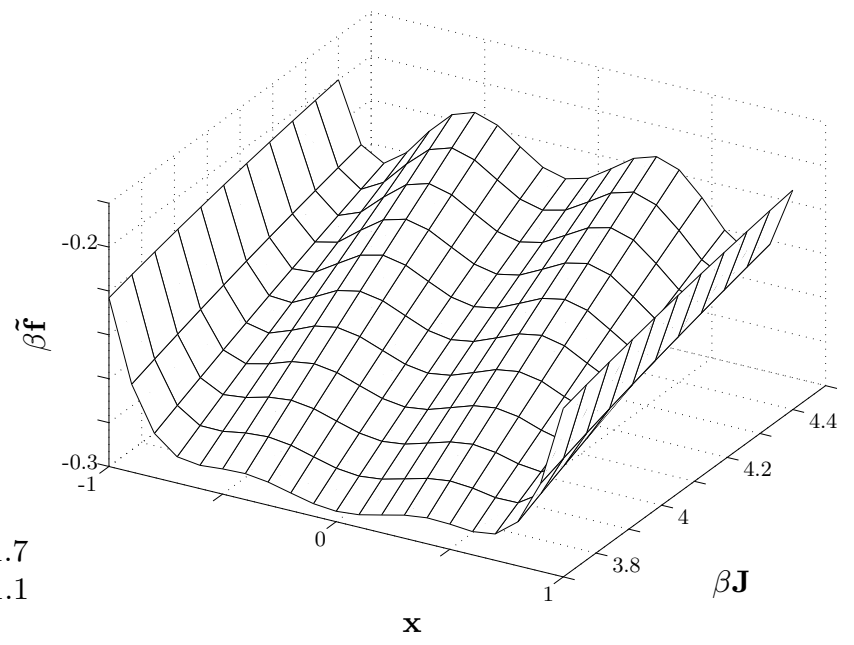

Fig. 4. $\beta \tilde{f}(\beta, x)$ as a function of $\beta J \in[3.7,4.4]$ and $x \in[-1,1]$ for $\Delta=0.476190$. The first order transition is at $\beta J=4$. Two side minima to $x=0$ first develop and then reach the same hight at the transition point.

Using Stirling's approximation in the large $N$ limit, the entropy, $S=k_{B} \ln \Omega$, is given by

$$
\begin{aligned}
S & =-k_{B} N\left[(1-q) \ln (1-q)+\frac{1}{2}(q+m) \ln (q+m)\right. \\
& \left.+\frac{1}{2}(q-m) \ln (q-m)-q \ln 2\right]
\end{aligned}
$$

where $q=Q / N$ and $m=M / N$ are the quadrupole moment and the magnetization per site, respectively. Let $\epsilon=E / \Delta N$ be the dimensionless energy per site, normalized by $\Delta$. Equation (11) may be written as

$$
q=\epsilon+K m^{2},
$$

where $K=J / 2 \Delta$. Using this relation, the entropy per site $s=S /\left(k_{B} N\right)$ can be expressed in terms of $m$ and $\epsilon$. At fixed $\epsilon$, the value of $m$ which maximizes the entropy corresponds to the equilibrium magnetization. The corresponding equilibrium entropy $s(\epsilon)=\max _{m} s(\epsilon, m)$ contains all the information about the thermodynamics of the system in the microcanonical ensemble. For instance, temperature can be obtained from the relation

$$
\frac{\Delta}{k_{B} T}=\frac{\partial s}{\partial \epsilon} .
$$

As usual in systems where the energy per particle is bounded from above, the model has both a positive and a negative temperature region: entropy is a one humped function of the energy. The interesting features take place in the positive temperature range. In order to locate the continuous transition line, one 
develops $s(\epsilon, m)$ in powers of $m$, in analogy with what has been done above for the canonical free energy

$$
s=s_{0}+A m^{2}+B m^{4}+O\left(m^{6}\right),
$$

where

$$
s_{0}=s(\epsilon, m=0)=-(1-\epsilon) \ln (1-\epsilon)-\epsilon \ln \epsilon+\epsilon \ln 2,
$$

and

$$
\begin{aligned}
& A=-K \ln \frac{\epsilon}{2(1-\epsilon)}-\frac{1}{2 \epsilon}, \\
& B=-\frac{K^{2}}{2 \epsilon(1-\epsilon)}+\frac{K}{2 \epsilon^{2}}-\frac{1}{12 \epsilon^{3}} .
\end{aligned}
$$

In the paramagnetic phase both $A$ and $B$ are negative, and the entropy is maximized by $m=0$. The continuous transition to the ferromagnetic phase takes place at $A=0$ for $B<0$. In order to obtain the critical line in the $(T, \Delta)$ plane we first observe that temperature is calculable on the critical line $(m=0)$ using (15) and (17)

$$
\frac{\Delta}{k_{B} T}=\ln \frac{2(1-\epsilon)}{\epsilon} .
$$

Requiring now that $A=0$, one gets the following expression for the critical line

$$
2 \bar{\beta} K=\frac{1}{2} e^{\bar{\beta}}+1,
$$

where $\bar{\beta} \equiv \beta \Delta$. Equivalently, this expression may be written as $\bar{\beta} K=1 / 2 \epsilon$. The microcanonical critical line thus coincides with the critical line (9) obtained for the canonical ensemble. The tricritical point of the microcanonical ensemble is obtained at $A=B=0$. Combining these equations with Eq. (19) one finds that at the tricritical point $\bar{\beta}$ satisfies

$$
\frac{1}{8 \bar{\beta}^{2}} \frac{e^{\bar{\beta}}+2}{e^{\bar{\beta}}}-\frac{1}{4 \bar{\beta}}+\frac{1}{12}=0 .
$$

Equations (20, 21) yield a tricritical point at $K \simeq 1.0813, \bar{\beta} \simeq 1.3998$. This has to be compared with the canonical tricritical point located at $K=3 / \ln (16) \simeq$ $1.0820, \bar{\beta}=\ln (4) \simeq 1.3995$. The two points, although very close to each other, do not coincide. The microcanonical critical line extends beyond the canonical one. In the region between the two tricritical points, the canonical ensemble yields a first order transition at a higher temperature, while in the microcanonical ensemble the transition is still continuous. It is in this region, as discussed below, that negative heat capacity appears. A schematic phase diagram near the canonical tricritical point (CTP) and the microcanonical one (MTP) is given in Fig. 同. Beyond the microcanonical tricritical point the temperature has a jump at the transition energy in the microcanonical ensemble. The two lines emerging on the right side from the MTP correspond to the two limiting temperatures 


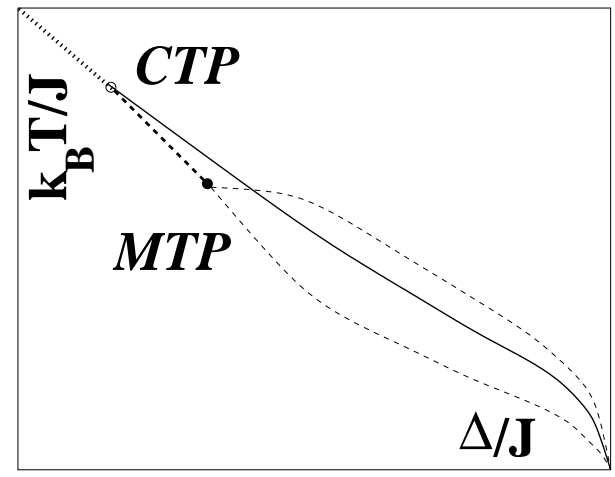

Fig. 5. A schematic representation of the phase diagram, where we expand the region around the canonical (CTP) and the microcanonical (MTP) tricritical points. The second order line, common to both ensembles, is dotted, the first order canonical transition line is solid and the microcanonical transition lines are dashed (with the bold dashed line representing a continuous transition)

which are reached when approaching the transition energy from below and from above (see Fig. 6d). The two microcanonical temperature lines and the canonical first-order transition line all merge on the $T=0$ line at $2 \Delta / J=1$.

To get a better undertanding of the microcanonical phase diagram and also to compare our results with those obtained for self-gravitating and for finite systems we consider the temperature-energy relation $T(\epsilon)$. This curve has two branches: a high energy branch (19) corresponding to $m=0$, and a low energy branch obtained from (15) using the spontaneous magnetization $m_{s}(\epsilon)$. At the intersection point of the two branches the two entropies become equal. Their first derivatives at the crossing point can be different, resulting in a jump in the temperature, i.e. a microcanonical first order transition. When the transition is continuous in the microcanonical ensemble, i.e. the first derivative of the entropy branches at the crossing point are equal, our model always displays, at variance with what happens for gravitational systems, a discontinuity in the second derivative of the entropy. This is due to the fact that here we have a true symmetry breaking transition. Fig. 6 displays the $T(\epsilon)$ curve for increasing values of $\Delta$. For $\Delta / J=\ln (4) / 3$ ), corresponding to the canonical tricritical point, the lower branch of the curve has a zero slope at the intersection point (Fig. 6a). Thus, the specific heat of the ordered phase diverges at this point. This effect signals the canonical tricritical point as it appears in the microcanonical ensemble. Increasing $\Delta$ to the region between the two tricritical points a negative specific heat in the microcanonical ensemble first arises $(\partial T / \partial \epsilon<0)$, see Fig. 6b. At the microcanonical tricritical point $\Delta$ the derivative $\partial T / \partial \epsilon$ of the lower branch diverges at the transition point, yielding a vanishing specific heat (Fig. 6ic). For larger values of $\Delta$ a jump in the temperature appears at the transition energy (Fig. 6d). The lower temperature corresponds to the $m=0$ solution (19) and the upper one is given by $\exp (\bar{\beta})=2\left(1-q^{*}\right) / \sqrt{\left(q^{*}\right)^{2}-\left(m^{*}\right)^{2}}$, where $m^{*}, q^{*}$ 
are the values of the order parameters of the ferromagnetic state at the transition energy. The negative specific heat branch disappears at larger values of $\Delta$, leaving just a temperature jump (see Fig. 6e). In the $\Delta / J \rightarrow 1 / 2$ limit the low temperature branch, corresponding to $q=m=1$ in the limit, shrinks to zero and the $m=0$ branch (19) occupies the full energy range (Fig. 6f).

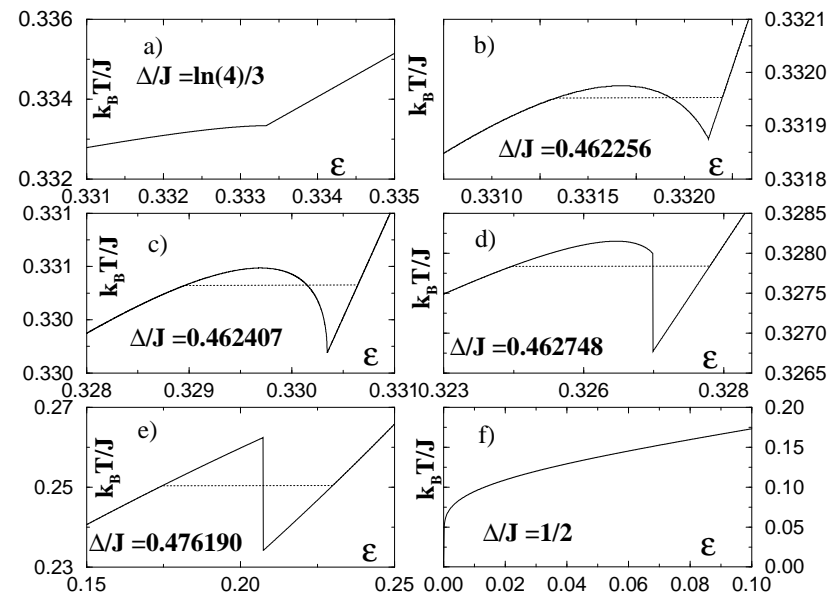

Fig. 6. The temperature-energy relation in the microcanonical ensemble for different values of $\Delta$. The dotted horizontal line in some of the plots is the Maxwell construction in the canonical ensemble and identifies the canonical first order transition temperature at the point where two minima of the free energy coexist. The vertical line in panels d) and e) marks the presence of a temperature jump: for this energy two entropy maxima of the same height coexist

We now present some general considerations concerning the intricate relation between canonical and microcanonical ensembles. The following discussion is quite general, and not specific to the BEG model. Let us first calculate the canonical partition sum using the density of states $\Omega=e^{N s(e, m)}$, where $e=E / N$ is the energy density and $m$ the order parameter (for the BEG model $e=\epsilon \Delta$, $m$ is the magnetization and $q$ is obtained from $e$ and $m$ by relation (14)).

$$
\begin{aligned}
Z & =\sum_{\left[S_{i}\right]} \exp \left(-\beta H\left(S_{i}\right)\right) \\
& =\sum_{[e, m]} e^{N s(e, m)} e^{-N \beta e},
\end{aligned}
$$

where we have replaced the sum over the configurations by the sum over the values of the energy per spin and the order parameter, which for finite $N$ take discrete values. In the thermodynamic limit $N \rightarrow \infty$, the free energy in the 
canonical ensemble is thus given by

$$
f(\beta)=\min _{e, m}\left(e-\frac{s(e, m)}{\beta}\right) .
$$

The point where the minimum is reached yields the mean energy and magnetization per spin in the canonical ensemble. As explained above, the microcanonical entropy is

$$
s(e)=\max _{m} s(e, m)
$$

and the magnetization in the microcanonical ensemble is the point where the maximum is reached.

All questions are now reduced to the solution of the two variational problems (23) and (24). The first order conditions are the same for the two problems

$$
\begin{aligned}
& \frac{\partial s}{\partial m}=0 \\
& \frac{\partial s}{\partial e}=\beta
\end{aligned}
$$

where the second condition is indeed given in the microcanonical case by the definition of temperature. We will denote by $e^{*}(\beta), m^{*}(\beta)$ the solution of the variational problem (25). Using (25), it is straightforward to verify that

$$
\frac{d(\beta f)}{d \beta}=e^{*}(\beta)
$$

meaning that the canonical mean energy coincides with the minimizing energy. Hence, the extrema in (23) and in (24) are the same. However this does not imply ensemble equivalence: we have to study the stability of these extrema.

In order to discuss the stability of the canonical solution one has to determine the sign of the eigenvalues of the Hessian of the function to be minimized in (23). The Hessian is

$$
\mathcal{H}=\frac{-1}{\beta}\left(\begin{array}{cc}
s_{m m} & s_{m e} \\
s_{\text {em }} & s_{\text {ee }}
\end{array}\right)
$$

where, for example, $s_{m m}$ is the second derivative of $s$ with respect to $m$. The extremum is a minimum if and only if the determinant and the trace of the Hessian are positive

$$
\begin{aligned}
-s_{e e}-s_{m m} & >0 \\
s_{e e} s_{m m}-s_{m e}^{2} & >0,
\end{aligned}
$$

which implies that $s_{e e}$ and $s_{m m}$ must be negative, and moreover $s_{e e}<-s_{m e}^{2} /\left|s_{m m}\right|$. This has strong implications on the canonical specific heat, which must be positive. Let us prove it using the variational approach, instead of the usual Thirring argument [4], that uses the expression of the canonical partition sum. Indeed, 
taking the derivatives of Eqs. (25) with respect to $\beta$, after having substituted into them $e^{*}(\beta), m^{*}(\beta)$, one gets

$$
\begin{aligned}
& s_{e e} \frac{d e^{*}}{d \beta}+s_{e m} \frac{d m^{*}}{d \beta}=1 \\
& s_{m e} \frac{d e^{*}}{d \beta}+s_{m m} \frac{d m^{*}}{d \beta}=0,
\end{aligned}
$$

where it is understood that all second derivatives are computed at $e^{*}(\beta), m^{*}(\beta)$. Recalling now that the specific heat per particle at constant volume is,

$$
\frac{C_{V}}{N k_{B}}=\frac{d e^{*}}{d T}=-\beta^{2} \frac{d e^{*}}{d \beta}
$$

one gets

$$
\frac{C_{V}}{N k_{B}}=\beta^{2} \frac{s_{m m}}{s_{e m}^{2}-s_{e e} s_{m m}},
$$

which is always positive if the stability conditions (29) are satisfied. However, since the stability condition in the microcanonical ensemble only requires that $s_{m m}$ is negative, in order to have an entropy maximum, this indirectly proves that a canonically stable solution is also microcanonically stable. The converse is not true: one may well have an entropy maximum, $s_{m m}<0$, which is a free energy saddle point, with $s_{e e}>0$. This implies that the specific heat (33) can be negative .

The above results are actually quite general, provided the canonical and microcanonical solutions are expressed through variational problems of the type (23) and (24). The extrema, and thus the caloric curves $T(e)$, are the same in the two ensembles, but the stability of the different branches is different (see also the important paper by Katz [12] and the contribution by Chavanis to this book [5] for a discussion of this point in connection to self-gravitating systems). Another example of this behavior is discussed for the HMF model in this book [16].

\section{Maxwell constructions}

In this Section we briefly comment on the Maxwell construction leading to temperature jumps in the microcanonical ensemble. This is quite a new feature, a sort of analogue of the latent heat phenomenon in the canonical ensemble.

Let us begin with the ordinary Maxwell construction in the canonical ensemble. We consider the curve $\beta(e)$ as in Fig. 7a. It is the situation of Fig. 6 $\mathrm{c}$, if for simplicity we disregard the discontinuity in the temperature derivative, which is irrelevant for the present reasoning. The equal area Maxwell condition $A_{1}=A_{2}$ simply reads

$$
\int_{e_{1}}^{e_{3}}\left[\beta(e)-\beta_{c}\right] d e=0,
$$

where $\beta_{c}$ is the first order transition inverse temperature. Since $\beta=d s / d e$, this implies

$$
s\left(e_{3}\right)-s\left(e_{1}\right)-\beta_{c}\left(e_{3}-e_{1}\right)=0 .
$$


Using now the definition of free energy, $f=e-s / \beta$, we obtain the usual equal free energy condition at a first order phase transition,

$$
f\left(e_{1}\right)=f\left(e_{3}\right) .
$$

Near a discontinuous microcanonical transition, the $\beta(e)$ curve has the typical shape given in Fig. $7 \mathrm{~b}$. This is in fact the case of Fig. 6 , if one continues the low energy branch of $\beta(e)$ above the microcanonical transition energy, drawing the metastable and the unstable lines as well. We invert this relation to obtain $e(\beta)$ in the vicinity of $e_{c}$. Then, the equal areas condition reads

$$
\int_{\beta_{1}}^{\beta_{3}}\left[e(\beta)-e_{c}\right] d \beta=0 .
$$

using $e=d(\beta f) / d \beta$, we get

$$
\beta_{3} f\left(\beta_{3}\right)-\beta_{1} f\left(\beta_{1}\right)-e_{c}\left(\beta_{3}-\beta_{1}\right)=0,
$$

which implies

$$
s\left(\beta_{3}\right)=s\left(\beta_{1}\right) .
$$

This is the equal entropy condition at a microcanonical discontinuous transition. Eq. (37) is valid only for the simple configuration of Fig. $7 \mathrm{~b}$; for more complex curves one has to evaluate the areas more carefully (see also the discussion by Chavanis (5))
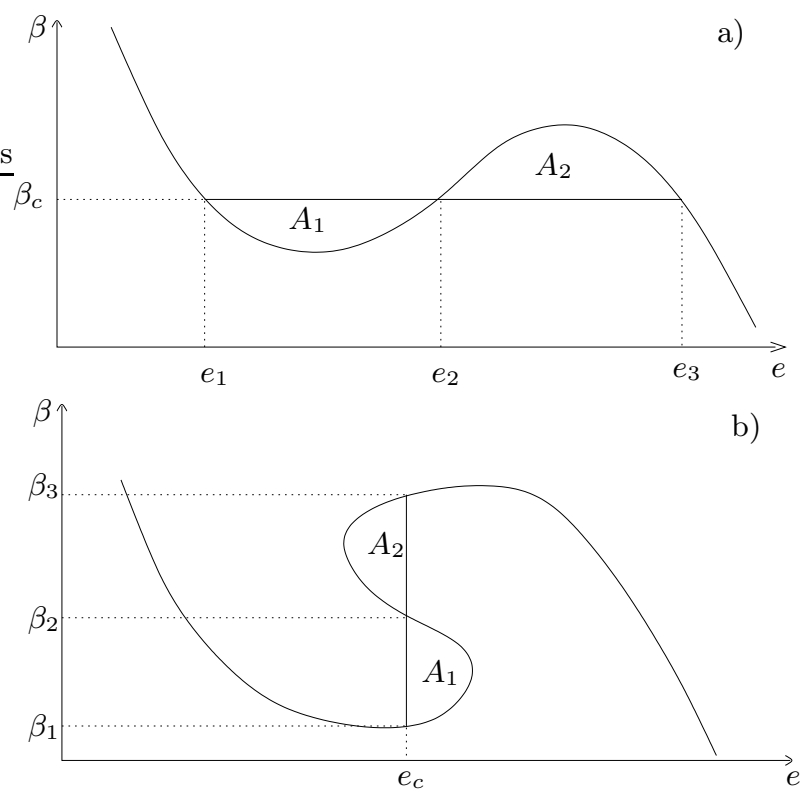

Fig. 7. Canonical (a) and microcanonical (b) Maxwell constructions. 


\section{The microcanonical solution by large deviation techniques}

In Section 3 we derived an expression for the logarithm of the density of states at fixed energy and magnetization, i.e. the entropy $s(e, m)$, using combinatorial techniques. We now present an alternative derivation of the entropy using Cramér's theorem [13]. This theorem, derived in the context of large deviation theory, states the following: consider a set of $N$ independent and identically distributed random variables in $R^{d},\left(X_{i}\right)_{i=1 \ldots N}$. One would like to know the probability distribution of the average $M_{N}=1 / N \sum X_{i}$. For this purpose, one defines, for $\lambda \in R^{d}$,

$$
\psi(\lambda)=\ln \left\langle e^{\lambda \cdot X}\right\rangle,
$$

where the average \langle\rangle is taken with respect to the common Probability distribution Function (PdF) of all $X_{i}$. Now let $s(x) / k_{B}$, with $x \in R^{d}$, be the inverse Legendre transform of $\psi$

$$
\frac{s(x)}{k_{B}}=-\sup _{\lambda \in R^{d}}(\lambda \cdot x-\psi(\lambda)) .
$$

Then, under quite general conditions, Cramér's theorem states that the PdF of the average is

$$
P\left(M_{N}=x\right) \sim e^{N \frac{s(x)}{k_{B}}} .
$$

This yields an expression for the logarithm of the density of states $s(x) / k_{B}$. The great advantage of this method is, of course, its generality, since it applies also when combinatorial tools are not available.

Let us give a brief heuristic argument for this result in the $d=1$ case, $\lambda, x \in R$. The probability of having $M_{N}=x$ is given by the volume in phase space compatible with $M_{N}=x$. Let $d \mu$ be the common measure of all $X_{i}$. One gets,

$$
\begin{aligned}
P\left(M_{N}=x\right) & =\int d \mu\left(X_{1}\right) \ldots d \mu\left(X_{N}\right) \delta\left(M_{N}-x\right) \\
& =\frac{1}{2 \pi i} \int_{\Gamma} d \lambda e^{-N \lambda x} \int d \mu\left(X_{1}\right) \ldots d \mu\left(X_{N}\right) e^{\lambda\left(X_{1}+\ldots+X_{N}\right)} \\
& =\frac{1}{2 \pi i} \int_{\Gamma} d \lambda e^{-N \lambda x}\left[\left\langle e^{\lambda X}\right\rangle\right]^{N}
\end{aligned}
$$

where the Dirac $\delta$ function has been represented by a Laplace integral over a path $\Gamma$ transverse to the real axis in the complex $\lambda$ plane. Evaluating the last integral using the saddle point method one is lead to look for the critical points of $\lambda x-\psi(\lambda)$, with $\psi(\lambda)=\ln \left[\left\langle e^{\lambda X}\right\rangle\right]$, which justifies Eq. (41).

As an example, let us apply this method to the BEG model' In this case the $X_{i}$ variables are bidimensional $(d=2), X_{i}=\left(S_{i}^{2}, S_{i}\right)$, so that

$$
\psi(\lambda, \rho)=\ln \left\langle e^{\lambda S_{i}^{2}+\rho S_{i}}\right\rangle
$$

1 An application to the Ising model and to other systems can be found in 14 


$$
=\ln \left(1+2 e^{\lambda} \cosh \rho\right)-\ln 3
$$

To calculate $s(q, m)$ we now have to solve equations $\partial \psi / \partial \lambda=q$ and $\partial \psi / \partial \rho=m$ for $\lambda$ and $\rho$. One gets

$$
\begin{aligned}
& \lambda=\ln \frac{q \sqrt{1-r^{2}}}{2(1-q)} \\
& \rho=\ln \sqrt{\frac{1+r}{1-r}}
\end{aligned}
$$

where $r$ is the ratio $m / q$. Substituting in (41), we obtain the expected result

$$
\frac{s(q, m)}{k_{B}}=-\frac{q+m}{2} \ln \frac{q+m}{2}-\frac{q-m}{2} \ln \frac{q-m}{2}-(1-q) \ln (1-q)-\ln 3,
$$

where the $-\ln 3$ constant is a normalization factor related to the fact that the total number of configurations is $3^{N}$.

\section{Slowly decaying interactions}

So far, we have restricted our study to infinite-range models. Space is in this case irrelevant, which simplifies very much the study. However, the physically interesting interactions (gravity for instance) are not infinite range, and one may wonder whether the properties of the previous Sections still hold in a more general case. To answer this question we discuss in this Section spin systems on $d$-dimensional lattices with pairwise interaction potential between two sites $i$ and $j$ decaying with the distance $r_{i j}$ like $1 / r_{i j}^{\alpha}(0 \leq \alpha<d)$, so that the interaction is long-range. An Ising model with short range algebraically decaying interactions $(\alpha>d)$ has been studied in the past by Dyson 15 . More recently, versions of the Hamiltonian Mean Field model [16], with slowly decaying long-range interactions among rotors $(\alpha<d)$ have been considered by several authors [17,18 and the problem has been discussed in a more general framework in Ref. $[19$.

Our main result is that all peculiar features of the microcanonical ensemble of infinite range models extend to these slowly decaying interactions. We proceed by considering a continuum, or coarse grained version of magnetic systems. We then explain that in fact these coarse grained models can be derived from the microscopic models, like the Ising or the BEG models considered in this paper, using the large deviations tools introduced in Section 5 (see [23] for a more thorough discussion of this point).

We begin by analyzing the phase diagram of the continuum version of the Ising model with algebraically decaying interactions $(0<\alpha<d)$. We show that as in the mean field model $(\alpha=0)$ this model does not exhibit a phase transition in the microcanonical ensemble. We then consider the continuum version of the BEG model.

The Ising Hamiltonian functional with algebraically decaying interactions in $d=1$ dimensions on the segment $[0,1]$ with periodic or free boundary conditions 
(to be discussed below) takes the form

$$
h_{\text {Ising }}=-\frac{\bar{J}}{2} \int_{0}^{1} d x \int_{0}^{1} d y \frac{m(x) m(y)}{r(x, y)^{\alpha}},
$$

where $m(x)$ is the local magnetization, $r(x, y)$ is the distance between $x$ and $y$ on the segment (the shortest distance for periodic boundary conditions), and $\bar{J}>0$ is a ferromagnetic coupling constant . The entropy density corresponding to a magnetization profile $m(x)$ of an Ising variable may be written as

$$
s_{\text {Ising }}=-k_{B} \int_{0}^{1} d x\left[\frac{(1+m)}{2} \ln \frac{(1+m)}{2}+\frac{(1-m)}{2} \ln \frac{(1-m)}{2}\right],
$$

Once the energy and entropy functionals are given, the microcanonical (canonical) solution is obtained by a maximization (minimization) of the entropy (freeenergy) functional under the constraint of constant energy (temperature). We begin below with the discussion of these two variational problems for the Ising model

$$
s_{\text {Ising }}(e)=\max _{m(x)}\left(s_{\text {Ising }}\left[(m(x)] \mid h_{\text {Ising }}=e\right),\right.
$$

in the microcanonical ensemble, and

$$
f_{\text {Ising }}(\beta)=\min _{m(x)}\left(h_{\text {Ising }}[m(x)]-\frac{1}{\beta} s_{\text {Ising }}[m(x)]\right)
$$

in the canonical ensemble .

So far, although we have stated the problem for the Ising model, we could have followed the same path, whatever the lattice model and the boundary conditions are. We can reach a general conclusion at this point by writing down the extremality conditions of the two variational problems (52) and (53). The first order reads

$$
\frac{\delta s_{\text {Ising }}}{\delta m(x)}=\beta \frac{\delta h_{\text {Ising }}}{\delta m(x)},
$$

in the microcanonical ensemble, where $\beta$ is a Lagrange multiplier. In the canonical ensemble

$$
\frac{\delta h_{I \operatorname{sing}}}{\delta m(x)}-\frac{1}{\beta} \frac{\delta s_{I \operatorname{sing}}}{\delta m(x)}=0 .
$$

Hence, the first order extremality conditions lead to the same equations for the two ensembles. However, this does not imply ensemble equivalence, since the stability of the solutions may differ in the two ensembles, as has been noted in Section 4 .

Before proceeding, let us briefly recall the results of Ref. [17]. These authors, discussing the Hamiltonian Mean Field model with slowly decaying interactions [16.22], remarked that, choosing appropriately the renormalization factor in the Hamiltonian, the thermodynamic behavior of the system turned out to be independent of $\alpha$; i.e. the system behaves at $0<\alpha<1$ (slowly decaying case) exactly as if $\alpha=0$ (mean-field case). An explanation of this result was 
provided by Campa et al. [18] through a canonical analysis of long-range interacting systems on lattices with periodic boundary conditions, and independently by Vollmayr-Lee and Luijten [19] in a more general context. Unfortunately, neither of the two latter methods provide the microcanonical solution. The method we present here is, instead, fully general and allows to discuss in detail how to extend the results for $\alpha=0$ to $\alpha>0$.

Let us solve now the variational problem of Eqs.(54) and (55) for the Ising model [21]. The magnetization profile that extremizes entropy and free energy satisfies the following self-consistency condition

$$
m(x)=\tanh \left(\beta \bar{J} \int_{0}^{1} \frac{m(y)}{r(x, y)^{\alpha}} d y\right)
$$

In the canonical ensemble, $\beta$ is fixed, whereas in the microcanonical ensemble it has to be tuned in order to get the required energy. For periodic boundary conditions, the integral on the r.h.s. of Eq. (56) does not depend on $x$, and Eq.56) always has a homogeneous solution with $m(x)$ independent of $x$. Eq. (56) then reduces to the usual consistency equation of the mean-field Ising model, and the system behaves exactly as in the $\alpha=0$ case, showing a phase-transition in the canonical ensemble (no transition is present in the microcanonical ensemble).

On the contrary, for free boundary conditions, there is no non-zero constant solution, and the system develops a non trivial magnetization profile that can be only numerically computed from Eq.(56). Moreover, in this case a phase transition is present in the canonical ensemble, while the microcanonical ensemble is always in a magnetically ordered phase. The magnetization profile in the microcanonical ensemble for free boundary conditions is shown in Fig. \& for three different values of $\alpha$.

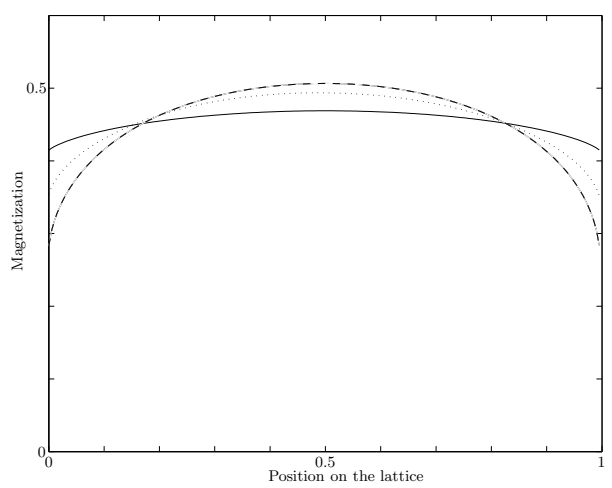

Fig. 8. Magnetization profile for the Ising model with slowly decaying interactions in the microcanonical ensemble. The energy density is fixed at $e=-0.1$ while $\alpha$ varies: $\alpha=0.2$ (full line), $\alpha=0.5$ (dotted line), $\alpha=0.8$ (dashed line). The boundary conditions are free. 
Let us now discuss the behavior of the BEG model for slowly decaying interactions. The energy functional is

$$
h_{B E G}=\Delta \int_{0}^{1} d x q(x)-\frac{\bar{J}}{2} \int_{0}^{1} d x \int_{0}^{1} d y \frac{m(x) m(y)}{r(x, y)^{\alpha}},
$$

where all is defined as before and, moreover, $q(x)$ is the quadrupolar field in the continuum on the segment $[0,1]$ (obtained in an analogous way as for the magnetization $m(x))$. The entropy functional of the BEG model is obtained from expression (13) by replacing $m, q$ by $m(x), q(x)$.

The BEG model is much richer, as has already been demonstrated for $\alpha=0$. The analysis of the microcanonical phase diagram for periodic boundary conditions follows the same path as that presented above for the Ising model. It is straightforward to show that the constant profiles $m(x)=m^{*}, q(x)=q^{*}$, with $m^{*}$ and $q^{*}$ given by the solution of the $\alpha=0$ model, are solutions of the Eqs. (54) and (55) (where we replace the Ising subscript by the BEG one) also for $\alpha>0$. Moreover, detailed calculations [24] show that these solutions are local maxima of the entropy and local minima of the free energy. Thus one concludes that for periodic systems the entropy and free energy are independent of $\alpha$ and the conclusions reached for $\alpha=0$ are valid for $0<\alpha<d$. In particular, ensemble inequivalence can be extended to the case of long-range algebraically decaying interactions.

Let us briefly sketch these calculations. To verify that uniform profiles are not destabilized by modulations, it is convenient to develop $q(x)$ and $m(x)$ in Fourier series: $q(x)=\sum_{k} \tilde{q}_{k} u_{k}(x), m(x)=\sum_{k} \tilde{m}_{k} u_{k}(x)$, where $k$ ranges from $-\infty$ to $+\infty$ and $u_{k}(x)=\cos k x$ if $k \geq 0, u_{k}(x)=\sin k x$ if $k<0$.

The Fourier representation, due to the periodicity, diagonalizes the Hamiltonian. The energy may thus be written as

$$
\epsilon=\tilde{q}_{0}-\bar{K} \sum_{k=-\infty}^{+\infty} \lambda_{k} \tilde{m}_{k}^{2}
$$

Here $\lambda_{k}$ are the energy eigenvalues and $\bar{K}=2^{\alpha} \bar{J} / 2 \Delta(1-\alpha)$ is chosen such that the largest eigenvalue is unity $\lambda_{0}=1$ (this amounts to choose the prefactor $\tilde{N}$ in the Kac prescription, see below).

The free energy functional is

$$
f[q, m]=\epsilon-1 / \beta \int s[q(x), m(x)] d x,
$$

so that we get the first order conditions

$$
\begin{aligned}
& \delta_{k 0} \beta-\int s_{q}[q, m] u_{k}(x) d x=0 \\
& -2 \beta \bar{K} \lambda_{k} \tilde{m}_{k}-\int s_{m}[q, m] u_{k}(x) d x=0
\end{aligned}
$$


We can easily check that the uniform profile $q=\tilde{q}_{0}, m=\tilde{m}_{0}$ (with $\tilde{q}_{0}$ and $\tilde{m}_{0}$ given by the $\alpha=0$ case) is indeed a solution of these conditions. To prove that the uniform profile is a free energy minimum, we evaluate now the Hessian matrix at this point

$$
\begin{aligned}
\frac{\partial f}{\partial \tilde{q}_{k} \partial \tilde{q}_{l}} & =-\frac{s_{q q}}{\beta} \delta_{k l} \\
\frac{\partial f}{\partial \tilde{q}_{k} \partial \tilde{m}_{l}} & =-\frac{s_{q m}}{\beta} \delta_{k l} \\
\frac{\partial f}{\partial \tilde{m}_{k} \partial \tilde{m}_{l}} & =-\frac{s_{m m}}{\beta} \delta_{k l}-2 \bar{K} \lambda_{k} \delta_{k l} .
\end{aligned}
$$

This infinite Hessian matrix turns out to be positive definite. To prove this, it is sufficient to verify that each matrix $H_{k}$

$$
H_{k}=-\left(\begin{array}{ll}
s_{q q} & s_{q m} \\
s_{q m} & s_{m m}+2 \bar{K} \beta \lambda_{k}
\end{array}\right)
$$

is positive definite, when taken at the solution point. This follows easily from the fact that $H_{0}$

$$
H_{0}=-\left(\begin{array}{ll}
s_{q q} & s_{q m} \\
s_{q m} & s_{m m}+2 \bar{K} \beta
\end{array}\right)
$$

is positive definite (because the point considered is the solution of the $\alpha=0$ case), and recalling that $\lambda_{k} \leq 1$ for whatever $k$.

We have thus proved that the local stability of the uniform profiles at $\alpha=0$ are not modified in the canonical ensemble when $\alpha>0$. All canonical $T(e)$ curves shown in Fig. 6 thus extend to the $\alpha>0$ case. We now make use of the general discussion at the end of Section 3. We recall that results there derived state, in particular, that a stable canonical solution is always a stable microcanonical one, and that if one follows a $T(e)$ curve, like in Fig. 6, a change of stability in the microcanonical ensemble may occur only where this curve has a vertical tangent (see [12] and the contribution by Chavanis [5] to this book). We thus conclude that the microcanonical part of Fig. 6 does not change either. This reasoning is however not rigorous, since we have considered only local stability; it may happen that when $\alpha>0$, a heterogeneous solution appears and it could yield the true free energy minimum or entropy maximum. Some numerical investigations we have carried out did not detect any such heterogeneous solution.

We now turn to a different issue, namely the correspondence between the microscopic models and the continuum ones. In the following we show that the Ising energy functional (50) can be derived by coarse graining from the microscopic Ising Hamiltonian with slowly decaying interactions

$$
H_{I s i n g}=-\frac{J}{2 \tilde{N}} \sum_{i, j} \frac{\sigma_{i} \sigma_{j}}{r_{i j}^{\alpha}},
$$

where $r_{i j}$ is the distance on a $1 \mathrm{D}$ lattice between spins at sites $i$ and $j$. The interaction is non integrable for $\alpha \leq 1$. The normalization $\tilde{N}=2^{\alpha} N^{1-\alpha} /(1-\alpha)$ 
ensures that the energy is extensive and the prefactor provides a convenient normalization for the eigenvalues of the Hamiltonian in the case of periodic boundary conditions. To make the correspondence between continuous and microscopic models explicit, one has to set $\bar{J}=(1-\alpha) J / 2^{\alpha}$ in Eq. 50 .

Analogously, the BEG microscopic model we refer to is

$$
H_{B E G}=\Delta \sum_{i=1}^{N} S_{i}^{2}-\frac{J}{2 \tilde{N}} \sum_{i, j} \frac{S_{i} S_{j}}{r_{i j}^{\alpha}} .
$$

The coarse-graining procedure we present, closely follows Ref. 20]. We will keep here a heuristic level of description, although all the following calculations can be made rigorous using the language and techniques of large deviation theory [13]. A more detailed account of this derivation is given in Ref. [23].

The first step involves a coarse-graining procedure. We divide the lattice in $N / n$ boxes, each one containing $n$ sites. We then describe macroscopically the system in the Ising case by an average magnetization in each box, or, in the BEG case, by the average $m$ and $q$. In the limit $N \rightarrow \infty, n \rightarrow \infty$ with $N / n \rightarrow \infty$, the system is then described by continuous functions $m(x), q(x)$, with $x \in[0,1]$ if the system length is normalized. We have to show in the following that all the information lost in the coarse graining procedure is unessential.

In the mean-field case $(\alpha=0)$, the Hamiltonian had a natural and exact expression as a function of the macroscopic parameters $m$ (Ising) or $m$ and $q$ (BEG). The long-range Ising case has already been discussed [21] and it has been shown that the intra-box couplings can be neglected in the thermodynamic limit, leading to the expression for the Hamiltonian functional (50) and for the entropy (51) as a function of the macroscopic parameter $m(x)$. The generalization to the BEG model of the method applied in Ref. 21] is straightforward and allows one to obtain the Hamiltonian functional (57). The estimation is uniform over all the microscopic configurations [23], and $r(x, y)$ is the distance between the two boxes located in $x$ and $y$. On the contrary, if $\alpha>d$, there is no way to approximate the Hamiltonian in the continuum limit described above as a functional of $m(x)$ and $q(x)$.

We show now how to construct the entropy functional for the Ising model. Its extension to the BEG model is straightforward. We have to estimate the probability to obtain a certain given macroscopic configuration $m(x)$, assuming the equiprobability of microscopic configurations. For a finite number of boxes, $N / n$, we can evaluate the probability to get a given average magnetization in each box

$$
\begin{aligned}
P\left(m_{1}, m_{2}, \ldots, m_{n}\right) & =P\left(m_{1}\right) P\left(m_{2}\right) \cdots P\left(m_{n}\right) \\
& \simeq e^{n s_{1}\left(m_{1}\right) / k_{B}} \cdots e^{n s_{n}\left(m_{n}\right) / k_{B}}
\end{aligned}
$$

where $s_{i}$ is the entropy associated to the $i$-th box. Letting $n$ and $N / n$ go to infinity, we obtain

$$
P[m(x)] \simeq e^{\frac{N}{k_{B}}} \int_{0}^{1} s(m(x)) d x
$$


which defines the entropy functional $s_{\text {Ising }}$ of the Ising model. Let us remark again that large deviation techniques 13] give a precise and rigorous meaning to these calculations, and allow one to extend this type of derivation to all sort of lattice models. The field $q(x)$ of the BEG model can be treated in a similar way as $m(x)$ and one gets the BEG entropy functional similarly.

Chavanis 5 and, Cohen and Ispolatov [25] analyze in this book more realistic off-lattice systems, self-gravitating or interacting through a $1 / r^{\alpha}$ potential $(\alpha<3)$. They study the thermodynamic properties of these models through a mean-field approximation, and the phenomenology is pretty much the same as the one decribed here. Unfortunately, to see the analogies, one must cope with discrepancies in the "vocabulary". For instance, the microcanonical first order transition described here is called gravitational first order transition, whereas the canonical first order one is called normal first order transition. Within the conventions of this paper one can see that, varying a control parameter (the interaction $K$ for the BEG model, and a parameter controlling the short range cut-off in gravitational models) the system crosses over from a microcanonical first order transition to a microcanonical second order one (or no transition at all in the gravitational systems) associated with a canonical first order, and then recovers full ensemble equivalence, crossing the critical point.

All these off-lattices studies raise the question of the validity of the meanfield approximation, which is intimately related to the scaling with $N$ of the thermodynamic variables and potentials. In our case, the mean field treatment is fully valid in the thermodynamic limit, but the problem was avoided using the physically unjustified Kac prescription [1] in which the coupling constants are rescaled by $\tilde{N}$.

\section{Conclusions}

In this paper a detailed comparison is made between the canonical and the microcanonical phase diagrams of the spin-1 BEG model with mean field long range interactions. Since systems with long range interactions, and particularly mean field models, are non-additive the two ensembles need not be equivalent. The BEG model, for which both phase diagrams can be evaluated analytically, provides a convenient and interesting ground for studying the distinctions between the two ensembles. Although the model is rather simple, it exhibits a non-trivial phase diagram, which in the canonical ensemble consists of both a first order and a second order transition lines separated by a tricritical point. It is shown that the microcanonical phase diagram, while it yields the same thermodynamic behavior in the region where the canonical transition is second order, it differs considerably from the canonical one in the region where the transition is first order. In particular it exhibits a region with a negative specific heat, a tricritical point whose location differs from that of the canonical ensemble, and a first order line at which the two coexisting phases have different temperatures. The mechanisms which lead to these features are rather general, and thus they are 
expected to take place in other models with long range interactions within the microcanonical ensemble (see also [26,27]).

A straightforward generalization of these results to models with algebraically decaying interactions are considered. It is shown that the mean field phase diagram remains valid even for these interactions, as long as they are long range.

It would be of great interest to extend this study in the future and to analyze and compare canonical and microcanonical phase diagrams in more complex cases, where higher order multicritical points are present. Such studies may eventually lead to general rules which should be obeyed by microcanonical phase diagrams of systems with long range interactions, in analogy to the rules existing for systems with short range interactions, like the Gibbs phase rules, the Landau and Lifshitz symmetry rules for continuous transitions and others.

\section{Acknowledgements}

We would like to warmly thank our collaborators Freddy Bouchet and François Leyvraz for fruitful interactions. We thank Thierry Dauxois and Dieter Gross for very useful discussions. This work has been partially supported by the EU contract No. HPRN-CT-1999-00163 (LOCNET network) and the Région RhôneAlpes for the fellowship $\mathrm{N}^{\circ}$ 01-009261-01. This work is also part of the contract COFIN00 on Chaos and localization in classical and quantum mechanics.

\section{References}

1. M. Kac, G.E. Uhlenbeck and P.C. Hemmer, J. Math. Phys. 4, 216 (1963).

2. V.A. Antonov Vest. Leningrad Univ. 7, 135 (1962); Translation in IAU Symposium 113, 525 (1995).

3. D. Lynden-Bell and R. Wood, Mon. Not. R. Astron. Soc. 138, 495 (1968); D. Lynden-Bell, Physica A 263, 293 (1999).

4. W. Thirring, Z. Phys. 235, 339 (1970); P. Hertel and W. Thirring, Ann. of Phys. 63, 520 (1971).

5. P.H. Chavanis, Statistical mechanics of two-dimensional vortices and threedimensional stellar systems, in "Dynamics and Thermodynamics of Systems with Long Range Interactions", T. Dauxois, S. Ruffo, E. Arimondo, M. Wilkens Eds., Lecture Notes in Physics Vol. 602, Springer (2002), (in this volume)

6. T. Padhmanaban, Statistical mechanics of gravitating systems in static and cosmological backgrounds, in "Dynamics and Thermodynamics of Systems with Long Range Interactions", T. Dauxois, S. Ruffo, E. Arimondo, M. Wilkens Eds., Lecture Notes in Physics Vol. 602, Springer (2002), (in this volume)

7. D.H.E. Gross, Microcanonical thermodynamics: Phase transitions in "small" systems, (World Scientific, Singapore, 2000) and Thermo-Statistics or Topology of the Microcanonical Entropy Surface, in "Dynamics and Thermodynamics of Systems with Long Range Interactions", T. Dauxois, S. Ruffo, E. Arimondo, M. Wilkens Eds., Lecture Notes in Physics Vol. 602, Springer (2002), (in this volume)

8. R.M. Lynden-Bell, in Gravitational dynamics, O. Lahav, E. Terlevich and R.J. Terlevich (eds.), Cambridge Univ. Press (1996); R.M. Lynden-Bell, Mol. Phys. 86, 1353 (1995). 
9. P. Chomaz and F. Gulminelli Phase transitions in finite systems, in "Dynamics and Thermodynamics of Systems with Long Range Interactions", T. Dauxois, S. Ruffo, E. Arimondo, M. Wilkens Eds., Lecture Notes in Physics Vol. 602, Springer (2002), (in this volume)

10. M. Blume, V.J. Emery and R.B. Griffiths, Phys. Rev. A 4, 1071 (1971).

11. J. Barré, D. Mukamel and S. Ruffo, Phys. Rev. Lett. 87, 030601 (2001).

12. J. Katz, Mon. Not. R. Astr. Soc. 183, 765 (1978).

13. A. Dembo, O. Zeitouni, Large Deviations Techniques and Applications, (Springer, Berlin, 1998).

14. R. S. Ellis, Physica D 133, 106, (1999).

15. F.J. Dyson, Comm. Math. Phys., 12, 91 (1969).

16. T. Dauxois, V. Latora, A. Rapisarda, S. Ruffo and A. Torcini, The Hamiltonian Mean Field Model: from Dynamics to Statistical Mechanics and back, in "Dynamics and Thermodynamics of Systems with Long Range Interactions", T. Dauxois, S. Ruffo, E. Arimondo, M. Wilkens Eds., Lecture Notes in Physics Vol. 602, Springer (2002), (in this volume)

17. C. Anteneodo and C. Tsallis, Phys. Rev. Lett. 80, 5313 (1998); F. Tamarit and C. Anteneodo, Phys. Rev. Lett. 84, 208 (2000);

18. A. Campa, A. Giansanti and D. Moroni, Phys. Rev. E 62, 303 (2000) and Chaos Solitons and Fractals, 13, 407 (2002).

19. B.P. Vollmayr-Lee and E. Luijten, Phys. Rev. E 63, 031108 (2001) and Phys. Rev. Lett. 85, 470 (2000).

20. R. S. Ellis, K. Haven, B. Turkington, J. Stat. Phys. 101, 999 (2000).

21. J. Barré, Physica A, 305, 172 (2002).

22. C. Tsallis, A. Rapisarda, V. Latora and F. Baldovin, Nonextensivity: from lowdimensional maps to Hamiltonian systems, in "Dynamics and Thermodynamics of Systems with Long Range Interactions", T. Dauxois, S. Ruffo, E. Arimondo, M. Wilkens Eds., Lecture Notes in Physics Vol. 602, Springer (2002), (in this volume)

23. J. Barré and F. Bouchet, "Mean-Field justified by large deviations results in longrange interacting systems". Proceedings of the Confererence "Dynamics and thermodynamics of systems with long range interactions", Les Houches, France, February 18-22 2002, Eds. T. Dauxois, E. Arimondo, S. Ruffo, M. Wilkens, published on http://www.ens-lyon.fr/ tdauxois/procs02/

24. J. Barré, D. Mukamel and S. Ruffo, to be published.

25. E.G.D. Cohen, I. Ispolatov, Phase transitions in systems with $1 / r^{\alpha}$ attractive interactions, in "Dynamics and Thermodynamics of Systems with Long Range Interactions", T. Dauxois, S. Ruffo, E. Arimondo, M. Wilkens Eds., Lecture Notes in Physics Vol. 602, Springer (2002), (in this volume)

26. F. Leyvraz and S. Ruffo, J. Phys. A, 35, 285 (2002) and Physica A, 305, 58 (2002).

27. I. Ispolatov and E.G.D. Cohen, Physica A, 295, 475 (2001). 